Supporting Information

of

\title{
Transmetalation of Phenylplatinum(II) Complex.
}

\section{Isolation and Characterization of a Dinuclear Intermediate in Intermolecular} Phenyl Ligand Transfer

\author{
Yuji Suzaki and Kohtaro Osakada* \\ Chemical Resources Laboratory, Tokyo Institute of Technology, 4259 Nagatsuta, Midori-ku, \\ Yokohama 226-8503, Japan
}


(a)

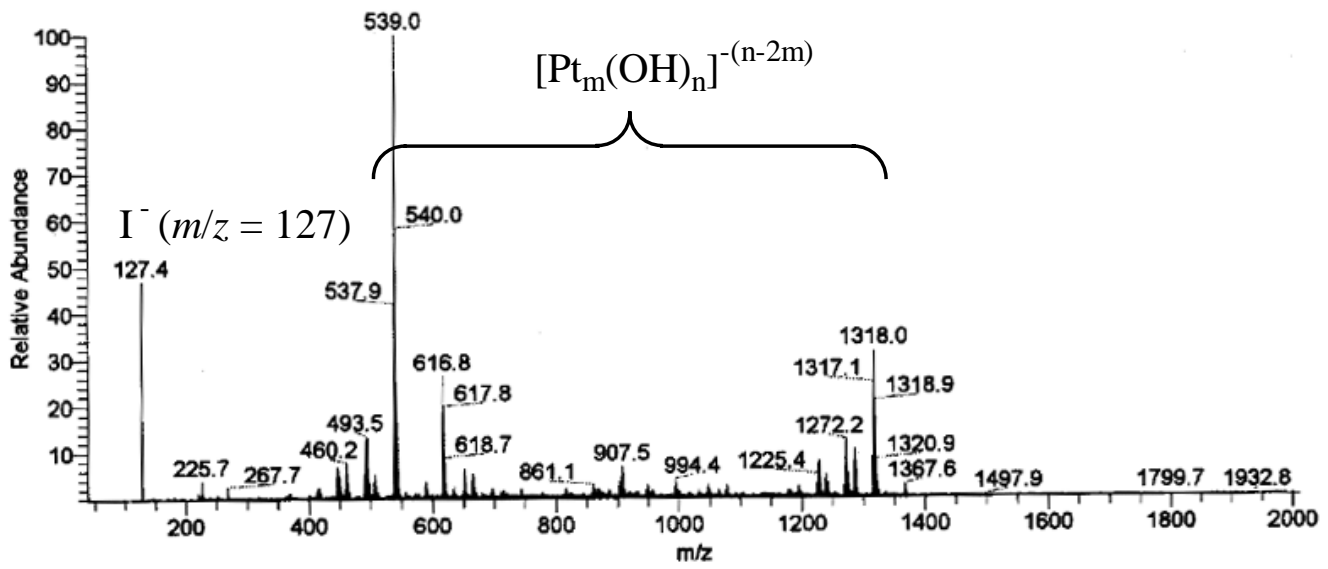

(b)

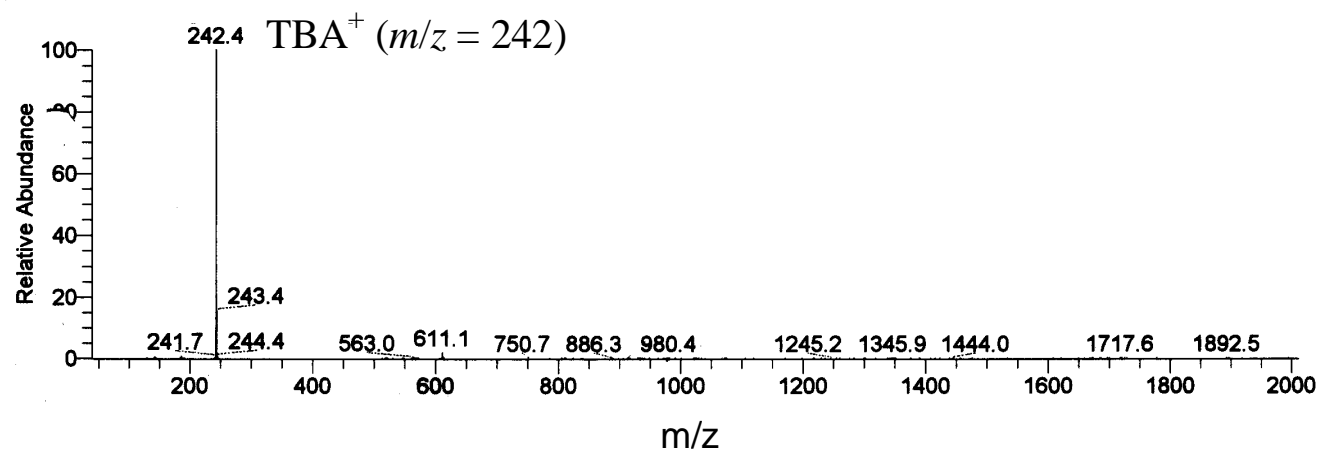

Figure S1. ESI/MS analysis of (a) anionic and (b) cationic fragments of oligomeric $\left[\mathrm{Pt}_{\mathrm{m}}(\mathrm{OH})_{\mathrm{n}}\right]^{-(\mathrm{n}-2 \mathrm{~m})}$. 


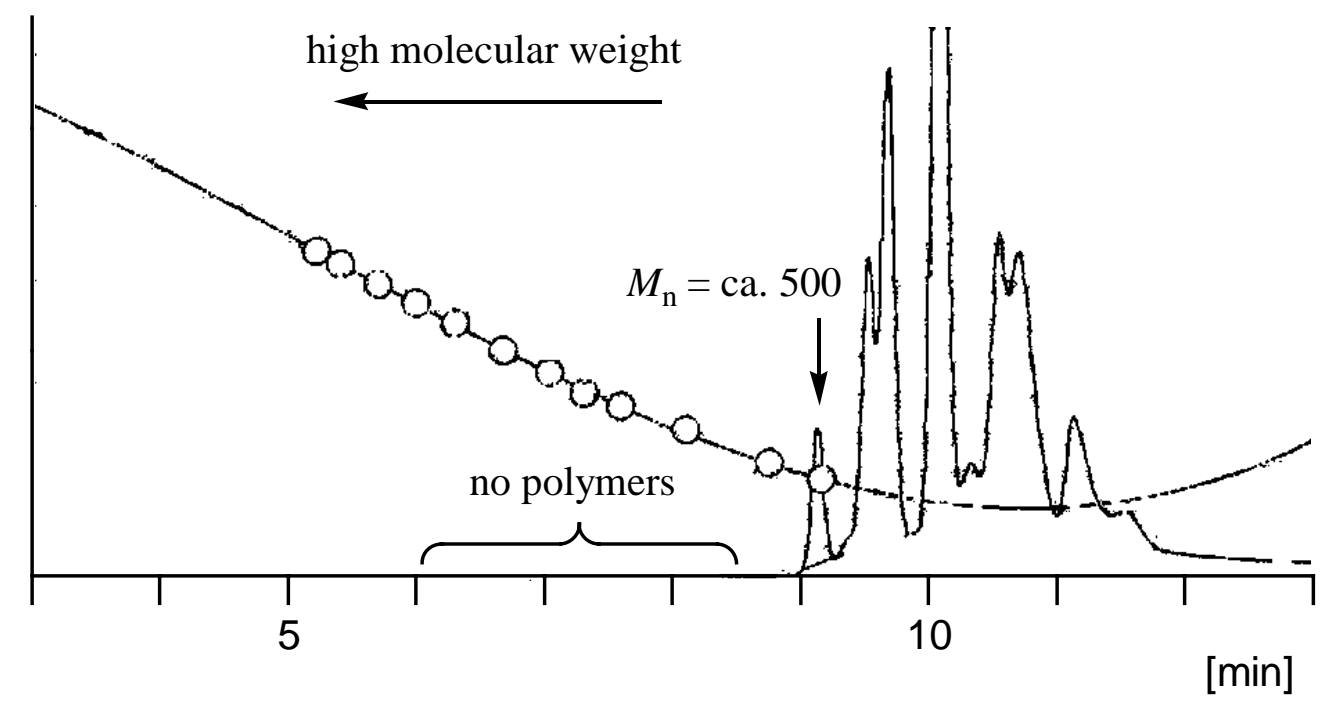

Figure S2. GPC (UV) analysis of oligomeric $\left[\mathrm{Pt}_{\mathrm{m}}(\mathrm{OH})_{\mathrm{n}}\right]^{-(\mathrm{n}-2 \mathrm{~m})}$. The molecular weight was determined in THF vs polystyrene standard.

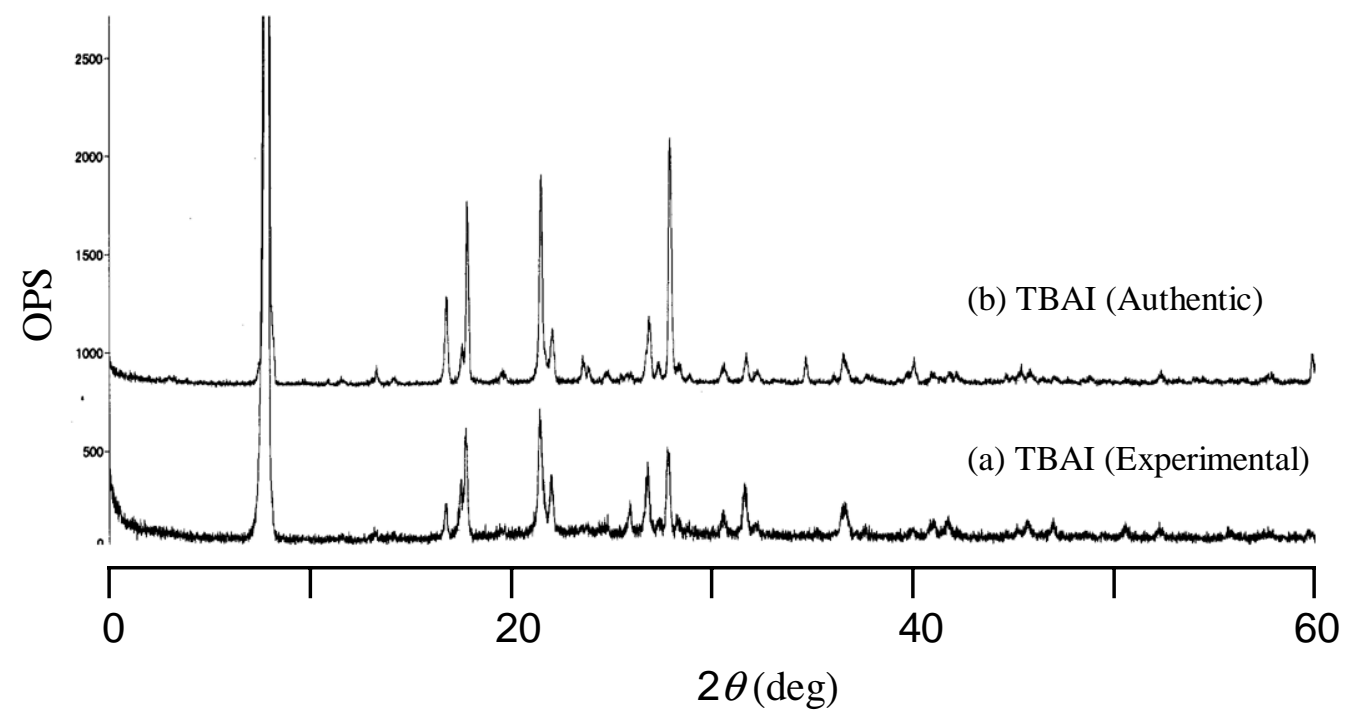

Figure S3. X-ray powder diffraction analysis of (a) TBAI obtained from the reaction mixture and (b) commercial available TBAI as an authentic sample. 
X-ray Structure Report for $1-\mathrm{BF}_{4}^{-}$

Data Collection

\section{Experimental}

A colorless prism crystal of $\mathrm{C}_{56} \mathrm{H}_{70} \mathrm{~B}_{2} \mathrm{~F}_{8} \mathrm{O}_{2} \mathrm{Pt}_{4}$ having approximate dimensions of $0.15 \times 0.15 \mathrm{x}$ $0.10 \mathrm{~mm}$ was mounted on a glass fiber. All measurements were made on a Rigaku Saturn CCD area detector with graphite monochromated Mo-K $\alpha$ radiation.

Indexing was performed from 0 images that were exposed for 0 seconds. The crystal-to-detector distance was $44.99 \mathrm{~mm}$.

Cell constants and an orientation matrix for data collection corresponded to a primitive monoclinic cell with dimensions:

$$
\begin{aligned}
& \mathrm{a}=10.5185(11) \AA \\
& \mathrm{b}=12.548(1) \AA \quad \AA \quad \beta=102.507(2)^{\mathrm{O}} \\
& \mathrm{c}=20.266(2) \AA \\
& \mathrm{V}=2611.4(5) \AA^{3}
\end{aligned}
$$

For $Z=2$ and F.W. $=1729.14$, the calculated density is $2.20 \mathrm{~g} / \mathrm{cm}^{3}$. Based on the systematic absences of:

$0 k 0: k \pm 2 n$

packing considerations, a statistical analysis of intensity distribution, and the successful solution and refinement of the structure, the space group was determined to be:

$$
\mathrm{P} 2{ }_{1}(\# 4)
$$

The data were collected at a temperature of $-160 \pm 1^{\circ} \mathrm{C}$ to a maximum $2 \theta$ value of $55.0^{\circ}$. A total of 720 oscillation images were collected. A sweep of data was done using $\omega$ scans from -110.0 to $70.0^{\circ}$ in $0.5^{\circ} \mathrm{step}$, at $\chi=45.0^{\circ}$ and $\phi=0.0^{\circ}$. The exposure rate was 40.0 [sec./0]. The detector swing angle was $-20.30^{\circ}$. A second sweep was performed using $\omega$ scans from -110.0 to $70.0^{\circ}$ in $0.5^{\circ}$ step, at $\chi=45.0^{\circ}$ and $\phi=90.0^{\circ}$. The exposure rate was 40.0 [sec./O]. The detector swing angle was $-20.30^{\circ}$. The crystal-to-detector distance was $44.99 \mathrm{~mm}$. Readout was performed in the $0.547 \mathrm{~mm}$ pixel mode.

\section{Data Reduction}

Of the 37804 reflections that were collected, 6031 were unique $\left(R_{\text {int }}=0.032\right)$; equivalent reflections were merged. Data were collected and processed using CrystalClear (Rigaku). Net intensities and sigmas were derived as follows:

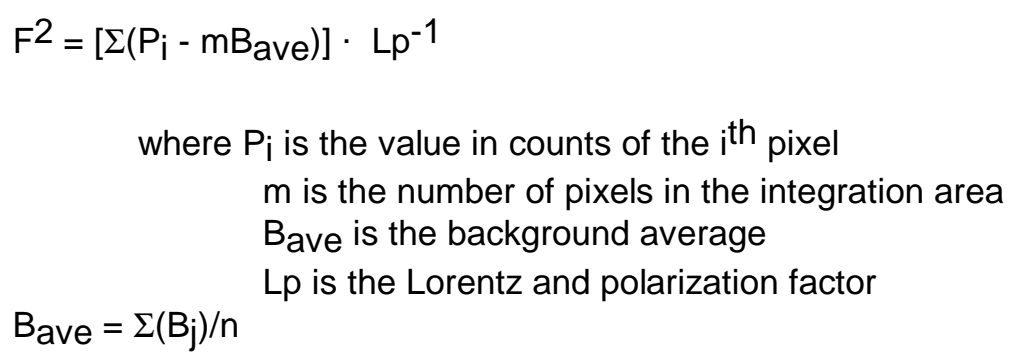

where $\mathrm{n}$ is the number of pixels in the background area

$B_{j}$ is the value of the $j^{\text {th }}$ pixel in counts 


$$
\begin{gathered}
\sigma^{2}\left(F^{2} h k l\right)=\left[\left(\Sigma P_{i}\right)+m\left(\left(\Sigma\left(B_{a v e}-B_{j}\right)^{2}\right) /(n-1)\right)\right] \cdot L p \cdot \text { errmul }+\left(\text { erradd } \cdot F^{2}\right)^{2} \\
\text { where erradd }=0.00 \\
\text { errmul }=1.00
\end{gathered}
$$

The linear absorption coefficient, $\mu$, for Mo-K $\alpha$ radiation is $107.1 \mathrm{~cm}^{-1}$. was applied which resulted in transmission factors ranging from 0.00 to 0.00 . The data were corrected for Lorentz and polarization effects.

\section{Structure Solution and Refinement}

The structure was solved by direct methods ${ }^{2}$ and expanded using Fourier techniques ${ }^{3}$. The non-hydrogen atoms were refined anisotropically. Hydrogen atoms were refined using the riding model. The final cycle of full-matrix least-squares refinement ${ }^{4}$ on $\mathrm{F}$ was based on 5882 observed reflections ( $\mathrm{I}>$ $3.00 \sigma(\mathrm{I}))$ and 719 variable parameters and converged (largest parameter shift was 0.09 times its esd) with unweighted and weighted agreement factors of:

$$
\begin{gathered}
\mathrm{R}=\Sigma\|\mathrm{Fo}|-| \mathrm{Fc}\| / \Sigma|\mathrm{Fo}|=0.029 \\
\mathrm{R}_{\mathrm{W}}=\left[\Sigma \mathrm{w}(|\mathrm{Fo}|-|\mathrm{Fc}|)^{2} / \Sigma \mathrm{w} \mathrm{Fo}^{2}\right]^{1 / 2}=0.041
\end{gathered}
$$

The standard deviation of an observation of unit weight ${ }^{5}$ was 1.01. A Sheldrick weighting scheme was used. Plots of $\Sigma \mathrm{w}(|\mathrm{Fo}|-|\mathrm{Fc}|)^{2}$ versus $|\mathrm{Fo}|$, reflection order in data collection, $\sin \theta / \lambda$ and various classes of indices showed no unusual trends. The maximum and minimum peaks on the final difference Fourier map corresponded to 8.57 and $-5.83 \mathrm{e}^{-} / \AA^{3}$, respectively.

Neutral atom scattering factors were taken from Cromer and Waber 6 . Anomalous dispersion effects were included in Fcalc 7 ; the values for $\Delta \mathrm{f}^{\prime}$ and $\Delta \mathrm{f}^{\prime \prime}$ were those of Creagh and McAuley ${ }^{8}$. The values for the mass attenuation coefficients are those of Creagh and Hubbell ${ }^{9}$. All calculations were performed using the CrystalStructure ${ }^{10,11}$ crystallographic software package.

\section{References}

(1) CrystalClear: Rigaku Corporation, 1999. CrystalClear Software User's Guide, Molecular Structure Corporation, (c) 2000.J.W.Pflugrath (1999) Acta Cryst. D55, 1718-1725.

(2) SIR92: Altomare, A., Cascarano, G., Giacovazzo, C., Guagliardi, A., Burla, M., Polidori, G., and Camalli, M. (1994) J. Appl. Cryst., 27, 435.

(3) DIRDIF99: Beurskens, P.T., Admiraal, G., Beurskens, G., Bosman, W.P., de Gelder, R., Israel, R. and Smits, J.M.M.(1999). The DIRDIF-99 program system, Technical Report of the Crystallography Laboratory, University of Nijmegen, The Netherlands.

(4) Least Squares function minimized:

$$
\Sigma w\left(\left|\mathrm{~F}_{\mathrm{O}}\right|-\left|\mathrm{F}_{\mathrm{C}}\right|\right)^{2} \quad \text { where } \mathrm{w}=\text { Least Squares weights. }
$$

(5) Standard deviation of an observation of unit weight:

$$
\begin{aligned}
& {\left[\Sigma w\left(\left|F_{\mathrm{o}}\right|-\left|\mathrm{F}_{\mathrm{C}}\right|\right)^{2} /\left(\mathrm{N}_{\mathrm{O}}-\mathrm{N}_{\mathrm{V}}\right)\right]^{1 / 2}} \\
& \text { where: } \mathrm{N}_{\mathrm{O}}=\text { number of observations }
\end{aligned}
$$

$$
\mathrm{N}_{\mathrm{V}} \text { = number of variables }
$$

(6) Cromer, D. T. \& Waber, J. T.; "International Tables for X-ray Crystallography", Vol. IV, The Kynoch Press, Birmingham, England, Table 2.2 A (1974).

(7) Ibers, J. A. \& Hamilton, W. C.; Acta Crystallogr., 17, 781 (1964).

(8) Creagh, D. C. \& McAuley, W.J .; "International Tables for Crystallography", Vol C, (A.J.C. Wilson, ed.), Kluwer Academic Publishers, Boston, Table 4.2.6.8, pages 219-222 (1992). 
(9) Creagh, D. C. \& Hubbell, J.H..; "International Tables for Crystallography", Vol C, (A.J.C. Wilson, ed.), Kluwer Academic Publishers, Boston, Table 4.2.4.3, pages 200-206 (1992).

(10) CrystalStructure 3.5.1: Crystal Structure Analysis Package, Rigaku and Rigaku/MSC (2000-2003). 9009 New Trails Dr. The Woodlands TX 77381 USA.

(11) CRYSTALS Issue 10: Watkin, D.J., Prout, C.K. Carruthers, J.R. \& Betteridge, P.W. Chemical Crystallography Laboratory, Oxford, UK. (1996) 


\section{EXPERIMENTAL DETAILS}

A. Crystal Data

Empirical Formula
Formula Weight
Crystal Color, Habit
Crystal Dimensions
Crystal System
Lattice Type
Detector Position
Pixel Size
Lattice Parameters

Space Group

$Z$ value

Dcalc

$F_{000}$

$\mu(\operatorname{MoK} \alpha)$
$\mathrm{C}_{56} \mathrm{H}_{70} \mathrm{~B}_{2} \mathrm{~F}_{8} \mathrm{O}_{2} \mathrm{Pt}_{4}$

1729.14

colorless, prism

$0.15 \times 0.15 \times 0.10 \mathrm{~mm}$

monoclinic

Primitive

$44.99 \mathrm{~mm}$

$0.137 \mathrm{~mm}$

$a=10.5185(11) \AA$

$\mathrm{b}=12.548(1) \AA$

$\mathrm{c}=20.266(2) \AA$

$\beta=102.507(2){ }^{\circ}$

$\mathrm{V}=2611.4(5) \AA^{3}$

P2 1 (\#4)

2

$2.199 \mathrm{~g} / \mathrm{cm}^{3}$

1632.00

$107.08 \mathrm{~cm}^{-1}$ 
B. Intensity Measurements

Detector

Goniometer

Radiation

Detector Aperture

Data Images

$\omega$ oscillation Range $(\chi=45.0, \phi=0.0)$

Exposure Rate

Detector Swing Angle

$\omega$ oscillation Range $(\chi=45.0, \phi=90.0)$

Exposure Rate

Detector Swing Angle

Detector Position

Pixel Size

$2 \theta_{\max }$

No. of Reflections Measured

Corrections
Rigaku Saturn

Rigaku AFC10

$\operatorname{MoK} \alpha(\lambda=0.71070 \AA)$

graphite monochromated

$70 \mathrm{~mm} \times 70 \mathrm{~mm}$

720 exposures

$-110.0-70.0^{\circ}$

$40.0 \mathrm{sec} . / \mathrm{O}$

$-20.30^{\circ}$

$-110.0-70.0^{\circ}$

$40.0 \mathrm{sec} . / 0$

$-20.30^{\circ}$

$44.99 \mathrm{~mm}$

$0.137 \mathrm{~mm}$

$55.0^{\circ}$

Total: 37804

Unique: $6031\left(R_{\text {int }}=0.032\right)$

Lorentz-polarization

Absorption

(trans. factors: $0.0000-0.0000$ ) 


\section{Structure Solution and Refinement}

\begin{tabular}{|c|c|}
\hline Structure Solution & Direct Methods (SIR92) \\
\hline Refinement & Full-matrix least-squares on $\mathrm{F}$ \\
\hline Function Minimized & $\Sigma w(|F o|-|F c|)^{2}$ \\
\hline Least Squares Weights & $1 /\left[0.0014 \mathrm{Fo}^{2}+1.0000 \sigma\left(\mathrm{Fo}^{2}\right)\right]$ \\
\hline Anomalous Dispersion & All non-hydrogen atoms \\
\hline No. Observations $(I>3.00 \sigma(I))$ & 5882 \\
\hline No. Variables & 719 \\
\hline Reflection/Parameter Ratio & 8.18 \\
\hline 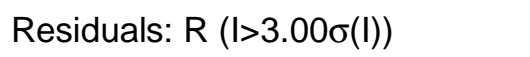 & 0.029 \\
\hline Residuals: Rw $(I>3.00 \sigma(I))$ & 0.041 \\
\hline Goodness of Fit Indicator & 1.012 \\
\hline Max Shift/Error in Final Cycle & 0.090 \\
\hline Maximum peak in Final Diff. Map & $8.57 \mathrm{e}^{-/ \AA^{3}}$ \\
\hline Minimum peak in Final Diff. Map & $-5.83 e^{-} / \AA^{3}$ \\
\hline
\end{tabular}


Table 1. Atomic coordinates and $\mathrm{Biso} / \mathrm{Beq}$

$\begin{array}{lllll}\text { atom } & \mathrm{x} & \mathrm{y} & \mathrm{z} & \mathrm{B} \text { eq } \\ \mathrm{Pt}(1) & 0.19438(3) & -0.30198(7) & -0.440440(10) & 0.932(6) \\ \mathrm{Pt}(2) & 0.41713(3) & -0.14476(7) & -0.50721(2) & 1.051(6) \\ \mathrm{Pt}(3) & 0.06988(3) & -0.26143(7) & -0.00569(2) & 0.855(6) \\ \mathrm{Pt}(4) & 0.35103(3) & -0.11318(7) & 0.063390(10) & 0.916(6) \\ \mathrm{F}(1) & 0.2784(9) & 0.3398(7) & 0.2989(4) & 4.4(2) \\ \mathrm{F}(2) & 0.2402(10) & 0.1648(7) & 0.2856(7) & 8.0(3) \\ \mathrm{F}(3) & 0.3079(12) & 0.232(2) & 0.3880(6) & 10.0(5) \\ \mathrm{F}(4) & 0.4464(8) & 0.2191(11) & 0.3246(5) & 6.7(3) \\ \mathrm{F}(5) & 0.8700(7) & 0.8874(6) & 0.1077(3) & 3.0(1) \\ \mathrm{F}(6) & 0.9159(6) & 0.7511(5) & 0.1804(3) & 2.49(13) \\ \mathrm{F}(7) & 0.9043(8) & 0.9185(6) & 0.2198(4) & 3.4(2) \\ \mathrm{F}(8) & 1.0761(6) & 0.8706(6) & 0.1726(3) & 2.9(1) \\ \mathrm{O}(1) & 0.3852(6) & -0.2707(5) & -0.4501(3) & 1.38(11) \\ \mathrm{O}(2) & 0.1535(6) & -0.1290(4) & 0.0517(3) & 1.04(11) \\ \mathrm{C}(1) & 0.1466(9) & -0.3248(7) & -0.5408(4) & 1.4(2) \\ \mathrm{C}(2) & 0.0828(10) & -0.2464(8) & -0.5838(5) & 1.9(2) \\ \mathrm{C}(3) & 0.0530(9) & -0.2614(8) & -0.6552(5) & 1.8(2) \\ \mathrm{C}(4) & 0.0816(10) & -0.3581(9) & -0.6824(5) & 2.1(2) \\ \mathrm{C}(5) & 0.1449(9) & -0.4375(8) & -0.6398(5) & 1.8(2) \\ \mathrm{C}(6) & 0.1800(9) & -0.4206(7) & -0.5687(4) & 1.4(2) \\ \mathrm{C}(7) & 0.2544(10) & -0.3215(8) & -0.3250(4) & 1.7(2) \\ \mathrm{C}(8) & 0.2099(10) & -0.2196(7) & -0.3359(4) & 1.6(2) \\ \mathrm{C}(9) & 0.0745(10) & -0.1854(8) & -0.3296(5) & 1.8(2) \\ \mathrm{C}(10) & -0.0222(10) & -0.1844(8) & -0.3991(5) & 1.7(2) \\ \mathrm{C}(11) & -0.0078(8) & -0.2773(8) & -0.4422(4) & 1.4(2) \\ \mathrm{C}(12) & 0.0262(9) & -0.3801(9) & -0.4251(5) & 1.9(2) \\ \mathrm{C}(13) & 0.0396(9) & -0.4228(7) & -0.3524(5) & 1.6(2) \\ \mathrm{C}(14) & 0.1812(9) & -0.4163(8) & -0.3088(4) & 1.5(2) \\ \mathrm{C}(15) & 0.3632(9) & -0.0424(8) & -0.4399(4) & 1.4(2) \\ \mathrm{C}(16) & 0.4533(10) & -0.0165(8) & -0.3797(5) & 1.7(2) \\ \mathrm{C}(17) & 0.4165(10) & 0.0455(8) & -0.3307(5) & 1.8(2) \\ \mathrm{C}(18) & 0.2903(10) & 0.0851(8) & -0.3406(5) & 1.9(2) \\ \mathrm{C}(19) & 0.1973(11) & 0.0566(8) & -0.3986(5) & 2.1(2) \\ \mathrm{C}(20) & 0.2377(9) & -0.0018(7) & -0.4486(4) & 1.4(2) \\ \mathrm{C}(21) & 0.3743(9) & -0.0224(7) & -0.5840(5) & 1.5(2) \\ \mathrm{C}(22) & 0.5058(10) & -0.0168(7) & -0.5531(5) & 1.6(2) \\ \mathrm{C}(23) & 0.6142(11) & -0.0547(8) & -0.5833(5) & 2.1(2) \\ & & & & \end{array}$


Table 1. Atomic coordinates and $\mathrm{Biso} / \mathrm{B}$ eq (continued)

\begin{tabular}{|c|c|c|c|c|}
\hline atom & $x$ & $y$ & z & $B_{e q}$ \\
\hline$C(24)$ & $0.6603(10)$ & $-0.1682(8)$ & $-0.5622(5)$ & $1.9(2)$ \\
\hline$C(25)$ & $0.5486(10)$ & $-0.2418(7)$ & $-0.5571(5)$ & $1.7(2)$ \\
\hline$C(26)$ & $0.4315(10)$ & $-0.2462(7)$ & $-0.5983(5)$ & $1.8(2)$ \\
\hline$C(27)$ & $0.3876(10)$ & $-0.1815(8)$ & $-0.6604(5)$ & $1.8(2)$ \\
\hline$C(28)$ & $0.3184(11)$ & $-0.0760(9)$ & $-0.6513(5)$ & $2.3(2)$ \\
\hline$C(29)$ & $0.1844(8)$ & $-0.3578(7)$ & $0.0585(4)$ & $0.9(2)$ \\
\hline$C(30)$ & $0.3008(9)$ & $-0.4021(7)$ & $0.0475(5)$ & $1.4(2)$ \\
\hline$C(31)$ & $0.3751(9)$ & $-0.4700(7)$ & $0.0945(5)$ & $1.8(2)$ \\
\hline$C(32)$ & $0.3439(9)$ & $-0.4950(7)$ & $0.1533(5)$ & $1.6(2)$ \\
\hline$C(33)$ & $0.2264(10)$ & $-0.4514(8)$ & $0.1661(5)$ & $1.8(2)$ \\
\hline$C(34)$ & $0.1493(9)$ & $-0.3831(7)$ & $0.1207(4)$ & $1.3(2)$ \\
\hline$C(35)$ & $-0.0299(9)$ & $-0.1484(7)$ & $-0.0931(5)$ & $1.5(2)$ \\
\hline$C(36)$ & $-0.1099(9)$ & $-0.1578(7)$ & $-0.0489(5)$ & $1.4(2)$ \\
\hline $\mathrm{C}(37)$ & $-0.2217(9)$ & $-0.2335(8)$ & $-0.0551(5)$ & $1.7(2)$ \\
\hline$C(38)$ & $-0.1962(10)$ & $-0.3439(7)$ & $-0.0817(5)$ & $1.8(2)$ \\
\hline$C(39)$ & $-0.0539(9)$ & $-0.3841(7)$ & $-0.0529(5)$ & $1.6(2)$ \\
\hline$C(40)$ & $0.0463(9)$ & $-0.3723(7)$ & $-0.0857(5)$ & $1.7(2)$ \\
\hline$C(41)$ & $0.0361(9)$ & $-0.3131(8)$ & $-0.1529(5)$ & $1.8(2)$ \\
\hline$C(42)$ & $-0.0430(10)$ & $-0.2069(9)$ & $-0.1601(6)$ & $2.3(2)$ \\
\hline$C(43)$ & $0.3211(9)$ & $-0.0941(8)$ & $-0.0393(5)$ & $1.6(2)$ \\
\hline$C(44)$ & $0.2670(9)$ & $0.0010(8)$ & $-0.0698(5)$ & $1.7(2)$ \\
\hline$C(45)$ & $0.2487(11)$ & $0.0126(9)$ & $-0.1404(5)$ & $2.5(2)$ \\
\hline$C(46)$ & $0.2842(13)$ & $-0.0642(10)$ & $-0.1785(5)$ & $3.0(2)$ \\
\hline$C(47)$ & $0.3414(13)$ & $-0.1569(10)$ & $-0.1493(6)$ & $3.0(3)$ \\
\hline$C(48)$ & $0.3568(12)$ & $-0.1721(8)$ & $-0.0788(5)$ & $2.3(2)$ \\
\hline$C(49)$ & $0.3788(10)$ & $-0.0706(9)$ & $0.1748(4)$ & $1.7(2)$ \\
\hline$C(50)$ & $0.4037(11)$ & $-0.1767(9)$ & $0.1699(5)$ & $2.5(2)$ \\
\hline$C(51)$ & $0.5443(13)$ & $-0.2228(10)$ & $0.1803(6)$ & $3.2(3)$ \\
\hline C(52) & $0.596(1)$ & $-0.2346(11)$ & $0.1164(7)$ & $3.6(3)$ \\
\hline C(53) & $0.5515(9)$ & $-0.1437(10)$ & $0.0681(7)$ & $2.8(2)$ \\
\hline C(54) & $0.5372(8)$ & $-0.0352(8)$ & $0.0786(5)$ & $1.5(2)$ \\
\hline$C(55)$ & $0.577(2)$ & $0.021(1)$ & $0.1449(6)$ & $4.8(4)$ \\
\hline$C(56)$ & $0.4807(10)$ & $0.0158(9)$ & $0.1915(5)$ & $2.1(2)$ \\
\hline$B(1)$ & $0.3216(12)$ & $0.2407(13)$ & $0.3203(8)$ & $3.6(3)$ \\
\hline$B(2)$ & $0.9421(10)$ & $0.8556(9)$ & $0.1707(5)$ & $1.6(2)$ \\
\hline
\end{tabular}

$B_{e q}=8 / 3 \pi^{2}\left(U_{11}\left(a a^{*}\right)^{2}+U_{22}\left(b b^{*}\right)^{2}+U_{33}\left(c c^{*}\right)^{2}+2 U_{12}\left(a a^{*} b b^{*}\right) \cos \gamma+2 U_{13}\left(a a^{*} c c^{*}\right) \cos \beta+2 U_{23}\left(b^{*} c c^{*}\right) \cos \alpha\right)$ 
Table 2. Anisotropic Displacement Parameters

\begin{tabular}{|c|c|c|c|c|c|c|}
\hline atom & $U_{11}$ & $U_{22}$ & $U_{33}$ & $\mathrm{U}_{12}$ & $U_{13}$ & $U_{23}$ \\
\hline Pt(1) & $0.0154(2)$ & $0.0097(1)$ & $0.0091(2)$ & $-0.00081(11)$ & $0.00007(11)$ & $0.00054(11)$ \\
\hline $\operatorname{Pt}(2)$ & $0.0182(2)$ & $0.0086(2)$ & $0.0131(2)$ & $-0.00036(11)$ & $0.00306(11)$ & $0.00038(11)$ \\
\hline $\operatorname{Pt}(3)$ & $0.0131(2)$ & $0.0067(1)$ & $0.0120(1)$ & $0.00024(11)$ & $0.00119(11)$ & $-0.00105(11)$ \\
\hline Pt(4) & $0.0126(2)$ & $0.0096(1)$ & $0.0117(2)$ & $-0.00104(10)$ & $0.00059(11)$ & $0.00072(10)$ \\
\hline$F(1)$ & $0.066(6)$ & $0.035(4)$ & $0.056(5)$ & $0.007(4)$ & $-0.008(4)$ & $-0.011(4)$ \\
\hline$F(2)$ & $0.063(6)$ & $0.028(4)$ & $0.177(12)$ & $-0.019(4)$ & $-0.058(7)$ & $0.028(6)$ \\
\hline$F(3)$ & $0.090(9)$ & $0.21(2)$ & $0.084(8)$ & $0.086(11)$ & $0.025(7)$ & $0.027(11)$ \\
\hline$F(4)$ & $0.039(5)$ & $0.141(12)$ & $0.061(6)$ & $0.037(6)$ & $-0.015(4)$ & $-0.040(7)$ \\
\hline$F(5)$ & $0.039(4)$ & $0.043(4)$ & $0.027(3)$ & $-0.004(3)$ & $-0.001(3)$ & $0.016(3)$ \\
\hline$F(6)$ & $0.034(3)$ & $0.021(3)$ & $0.039(3)$ & $0.003(3)$ & $0.005(3)$ & $0.005(3)$ \\
\hline$F(7)$ & $0.056(4)$ & $0.034(4)$ & $0.039(4)$ & $0.012(3)$ & $0.011(3)$ & $-0.016(3)$ \\
\hline$F(8)$ & $0.028(3)$ & $0.053(4)$ & $0.033(3)$ & $-0.013(3)$ & $0.016(3)$ & $-0.011(3)$ \\
\hline $\mathrm{O}(1)$ & $0.024(3)$ & $0.014(3)$ & $0.015(3)$ & $-0.002(2)$ & $0.005(2)$ & $0.008(2)$ \\
\hline $\mathrm{O}(2)$ & $0.014(3)$ & $0.009(3)$ & $0.019(3)$ & $-0.003(2)$ & $0.009(2)$ & $-0.007(2)$ \\
\hline$C(1)$ & $0.018(4)$ & $0.019(4)$ & $0.015(4)$ & $-0.008(3)$ & $-0.005(3)$ & $-0.004(3)$ \\
\hline$C(2)$ & $0.029(5)$ & $0.020(5)$ & $0.023(5)$ & $-0.008(4)$ & $0.007(4)$ & $0.003(4)$ \\
\hline$C(3)$ & $0.029(5)$ & $0.023(4)$ & $0.016(4)$ & $0.003(4)$ & $-0.001(3)$ & $0.004(4)$ \\
\hline$C(4)$ & $0.024(5)$ & $0.039(6)$ & $0.016(4)$ & $0.003(4)$ & $0.001(4)$ & $0.002(4)$ \\
\hline$C(5)$ & $0.023(5)$ & $0.027(5)$ & $0.018(4)$ & $-0.004(4)$ & $0.004(4)$ & $-0.006(4)$ \\
\hline$C(6)$ & $0.022(4)$ & $0.017(4)$ & $0.012(4)$ & $-0.002(3)$ & $0.001(3)$ & $0.001(3)$ \\
\hline$C(7)$ & $0.026(5)$ & $0.020(5)$ & $0.011(4)$ & $0.001(4)$ & $-0.008(3)$ & $0.002(3)$ \\
\hline $\mathrm{C}(8)$ & $0.036(5)$ & $0.010(4)$ & $0.013(4)$ & $-0.001(4)$ & $-0.002(4)$ & $-0.003(3)$ \\
\hline$C(9)$ & $0.032(5)$ & $0.021(4)$ & $0.015(4)$ & $-0.002(4)$ & $0.004(4)$ & $-0.001(3)$ \\
\hline$C(10)$ & $0.024(5)$ & $0.022(4)$ & $0.020(4)$ & $0.000(4)$ & $0.010(4)$ & $-0.004(4)$ \\
\hline$C(11)$ & $0.007(4)$ & $0.029(5)$ & $0.014(4)$ & $0.009(3)$ & $0.001(3)$ & $0.000(4)$ \\
\hline$C(12)$ & $0.016(4)$ & $0.027(5)$ & $0.027(5)$ & $-0.006(4)$ & $-0.004(4)$ & $-0.001(4)$ \\
\hline$C(13)$ & $0.022(5)$ & $0.015(4)$ & $0.024(5)$ & $-0.006(3)$ & $0.004(4)$ & $-0.001(3)$ \\
\hline$C(14)$ & $0.019(4)$ & $0.027(5)$ & $0.011(4)$ & $0.006(4)$ & $0.002(3)$ & $0.004(3)$ \\
\hline$C(15)$ & $0.025(4)$ & $0.017(4)$ & $0.011(4)$ & $-0.005(3)$ & $0.006(3)$ & $0.005(3)$ \\
\hline$C(16)$ & $0.025(5)$ & $0.017(4)$ & $0.020(4)$ & $0.001(3)$ & $0.004(4)$ & $-0.001(4)$ \\
\hline$C(17)$ & $0.029(5)$ & $0.019(4)$ & $0.016(4)$ & $0.002(4)$ & $-0.003(3)$ & $0.002(4)$ \\
\hline$C(18)$ & $0.031(5)$ & $0.018(4)$ & $0.024(5)$ & $0.005(4)$ & $0.010(4)$ & $0.000(4)$ \\
\hline$C(19)$ & $0.034(6)$ & $0.023(5)$ & $0.032(5)$ & $0.005(4)$ & $0.023(4)$ & $-0.002(4)$ \\
\hline$C(20)$ & $0.021(4)$ & $0.019(4)$ & $0.015(4)$ & $-0.002(3)$ & $0.005(3)$ & $0.003(3)$ \\
\hline$C(21)$ & $0.031(5)$ & $0.006(4)$ & $0.017(4)$ & $0.000(3)$ & $0.003(4)$ & $0.001(3)$ \\
\hline C(22) & $0.028(5)$ & $0.014(4)$ & $0.018(4)$ & $-0.003(3)$ & $0.002(4)$ & $0.000(3)$ \\
\hline$C(23)$ & $0.040(6)$ & $0.017(4)$ & $0.023(5)$ & $-0.003(4)$ & $0.003(4)$ & $-0.001(4)$ \\
\hline
\end{tabular}




\section{S13}

Table 2. Anisotropic Displacement Parameters (continued)

\begin{tabular}{|c|c|c|c|c|c|c|}
\hline atom & $\mathrm{U}_{11}$ & $U_{22}$ & $U_{33}$ & $U_{12}$ & $U_{13}$ & $U_{23}$ \\
\hline$C(24)$ & $0.030(5)$ & $0.017(5)$ & $0.025(5)$ & $-0.003(4)$ & $0.006(4)$ & $-0.006(3)$ \\
\hline$C(25)$ & $0.030(5)$ & $0.012(4)$ & $0.024(4)$ & $0.005(4)$ & $0.012(4)$ & $-0.002(3)$ \\
\hline$C(26)$ & $0.033(5)$ & $0.010(4)$ & $0.030(5)$ & $-0.010(4)$ & $0.016(4)$ & $-0.010(4)$ \\
\hline $\mathrm{C}(27)$ & $0.024(5)$ & $0.027(5)$ & $0.019(4)$ & $-0.003(4)$ & $0.010(4)$ & $-0.003(4)$ \\
\hline$C(28)$ & $0.031(5)$ & $0.035(6)$ & $0.021(5)$ & $-0.003(5)$ & $0.002(4)$ & $0.006(4)$ \\
\hline$C(29)$ & $0.009(4)$ & $0.011(4)$ & $0.018(4)$ & $0.000(3)$ & $0.007(3)$ & $-0.001(3)$ \\
\hline$C(30)$ & $0.021(4)$ & $0.008(3)$ & $0.027(5)$ & $0.001(3)$ & $0.011(4)$ & $0.006(3)$ \\
\hline$C(31)$ & $0.017(4)$ & $0.010(4)$ & $0.038(5)$ & $-0.001(3)$ & $-0.001(4)$ & $-0.000(4)$ \\
\hline$C(32)$ & $0.023(5)$ & $0.013(4)$ & $0.022(4)$ & $0.000(3)$ & $-0.002(4)$ & $0.004(3)$ \\
\hline $\mathrm{C}(33)$ & $0.033(5)$ & $0.017(4)$ & $0.022(4)$ & $0.006(4)$ & $0.013(4)$ & $0.005(4)$ \\
\hline$C(34)$ & $0.026(5)$ & $0.018(4)$ & $0.011(4)$ & $-0.000(4)$ & $0.012(3)$ & $0.001(3)$ \\
\hline$C(35)$ & $0.022(4)$ & $0.011(4)$ & $0.022(4)$ & $0.000(3)$ & $-0.000(3)$ & $0.007(3)$ \\
\hline$C(36)$ & $0.015(4)$ & $0.012(4)$ & $0.023(4)$ & $0.011(3)$ & $-0.003(3)$ & $0.004(3)$ \\
\hline $\mathrm{C}(37)$ & $0.015(4)$ & $0.021(5)$ & $0.029(5)$ & $0.002(4)$ & $0.000(3)$ & $0.008(4)$ \\
\hline$C(38)$ & $0.026(5)$ & $0.014(4)$ & $0.024(5)$ & $-0.009(4)$ & $-0.004(4)$ & $-0.000(4)$ \\
\hline C(39) & $0.025(5)$ & $0.011(4)$ & $0.020(4)$ & $-0.001(3)$ & $-0.003(4)$ & $0.003(3)$ \\
\hline$C(40)$ & $0.026(5)$ & $0.018(4)$ & $0.018(4)$ & $0.002(4)$ & $-0.005(4)$ & $-0.018(4)$ \\
\hline $\mathrm{C}(41)$ & $0.018(4)$ & $0.030(5)$ & $0.021(4)$ & $-0.002(4)$ & $0.006(3)$ & $-0.011(4)$ \\
\hline $\mathrm{C}(42)$ & $0.020(5)$ & $0.034(6)$ & $0.033(5)$ & $-0.006(4)$ & $0.006(4)$ & $0.005(4)$ \\
\hline C(43) & $0.016(4)$ & $0.021(5)$ & $0.025(5)$ & $-0.002(4)$ & $0.006(3)$ & $0.002(4)$ \\
\hline $\mathrm{C}(44)$ & $0.019(4)$ & $0.025(5)$ & $0.019(4)$ & $-0.003(4)$ & $0.005(3)$ & $0.003(4)$ \\
\hline C(45) & $0.039(6)$ & $0.034(6)$ & $0.017(5)$ & $-0.017(5)$ & $-0.005(4)$ & $0.008(4)$ \\
\hline$C(46)$ & $0.061(8)$ & $0.039(6)$ & $0.013(4)$ & $-0.021(6)$ & $0.006(5)$ & $0.002(4)$ \\
\hline $\mathrm{C}(47)$ & $0.067(8)$ & $0.034(6)$ & $0.022(5)$ & $-0.023(6)$ & $0.027(5)$ & $-0.015(5)$ \\
\hline$C(48)$ & $0.055(7)$ & $0.021(5)$ & $0.015(4)$ & $-0.013(5)$ & $0.013(4)$ & $-0.001(4)$ \\
\hline C(49) & $0.024(5)$ & $0.034(5)$ & $0.009(4)$ & $0.001(4)$ & $0.008(3)$ & $-0.002(4)$ \\
\hline $\mathrm{C}(50)$ & $0.033(6)$ & $0.033(5)$ & $0.021(5)$ & $-0.016(5)$ & $-0.009(4)$ & $0.013(4)$ \\
\hline$C(51)$ & $0.062(8)$ & $0.030(6)$ & $0.026(5)$ & $0.015(6)$ & $0.001(5)$ & $0.005(5)$ \\
\hline C(52) & $0.047(8)$ & $0.036(7)$ & $0.056(8)$ & $-0.001(6)$ & $0.020(6)$ & $-0.011(6)$ \\
\hline C(53) & $0.004(4)$ & $0.039(6)$ & $0.062(8)$ & $0.010(4)$ & $0.003(4)$ & $0.005(6)$ \\
\hline C(54) & $0.013(4)$ & $0.028(5)$ & $0.016(4)$ & $-0.011(4)$ & $0.006(3)$ & $0.002(4)$ \\
\hline C(55) & $0.071(10)$ & $0.088(12)$ & $0.029(6)$ & $-0.063(10)$ & $0.022(6)$ & $-0.018(7)$ \\
\hline$C(56)$ & $0.021(5)$ & $0.026(5)$ & $0.029(5)$ & $-0.008(4)$ & $-0.002(4)$ & $-0.005(4)$ \\
\hline $\mathrm{B}(1)$ & $0.018(5)$ & $0.046(8)$ & $0.060(9)$ & $-0.011(6)$ & $-0.016(6)$ & $0.012(7)$ \\
\hline $\mathrm{B}(2)$ & $0.021(5)$ & $0.016(4)$ & $0.021(5)$ & $0.002(4)$ & $-0.001(4)$ & $-0.002(4)$ \\
\hline
\end{tabular}

The general temperature factor expression: $\exp \left(-2 \pi^{2}\left(a^{\star 2} U_{11} h^{2}+b^{* 2} U_{22} k^{2}+\left.c^{\star 2} U_{33}\right|^{2}+2 a^{*} b^{*} U_{12} h k+\right.\right.$ $\left.2 a^{*} c^{*} U_{13} h l+2 b^{*} c^{*} U_{23} k l\right)$ ) 
Table 3. Bond lengths $(\AA)$

\begin{tabular}{|c|c|c|c|c|c|}
\hline atom & atom & distance & atom & atom & distance \\
\hline $\mathrm{Pt}(1)$ & $\mathrm{O}(1)$ & $2.096(7)$ & $\mathrm{Pt}(1)$ & $C(1)$ & $2.008(9)$ \\
\hline $\operatorname{Pt}(1)$ & $C(7)$ & $2.300(8)$ & $\operatorname{Pt}(1)$ & $C(8)$ & $2.332(9)$ \\
\hline $\operatorname{Pt}(1)$ & $C(11)$ & $2.142(8)$ & $\operatorname{Pt}(1)$ & $C(12)$ & $2.10(1)$ \\
\hline $\operatorname{Pt}(2)$ & $O(1)$ & $2.030(6)$ & $\operatorname{Pt}(2)$ & $C(15)$ & $2.041(9)$ \\
\hline $\operatorname{Pt}(2)$ & $C(21)$ & $2.163(8)$ & $\operatorname{Pt}(2)$ & $C(22)$ & $2.17(1)$ \\
\hline $\mathrm{Pt}(2)$ & $C(25)$ & $2.24(1)$ & $\mathrm{Pt}(2)$ & $C(26)$ & $2.27(1)$ \\
\hline $\mathrm{Pt}(3)$ & $\mathrm{O}(2)$ & $2.108(6)$ & $\mathrm{Pt}(3)$ & $C(29)$ & $1.982(8)$ \\
\hline $\mathrm{Pt}(3)$ & $C(35)$ & $2.333(9)$ & $\mathrm{Pt}(3)$ & $C(36)$ & $2.306(8)$ \\
\hline $\mathrm{Pt}(3)$ & $C(39)$ & $2.107(9)$ & $\mathrm{Pt}(3)$ & $C(40)$ & $2.111(9)$ \\
\hline $\mathrm{Pt}(4)$ & $\mathrm{O}(2)$ & $2.049(6)$ & $\operatorname{Pt}(4)$ & $C(43)$ & $2.05(1)$ \\
\hline $\mathrm{Pt}(4)$ & $C(49)$ & $2.277(9)$ & $\operatorname{Pt}(4)$ & $C(50)$ & $2.25(1)$ \\
\hline $\mathrm{Pt}(4)$ & $C(53)$ & $2.12(1)$ & $\operatorname{Pt}(4)$ & $C(54)$ & $2.151(9)$ \\
\hline$F(1)$ & $\mathrm{B}(1)$ & $1.36(2)$ & $F(2)$ & $B(1)$ & $1.37(2)$ \\
\hline$F(3)$ & $B(1)$ & $1.41(2)$ & $F(4)$ & $B(1)$ & $1.32(2)$ \\
\hline$F(5)$ & $\mathrm{B}(2)$ & $1.39(1)$ & $F(6)$ & $B(2)$ & $1.36(1)$ \\
\hline$F(7)$ & $B(2)$ & $1.39(1)$ & $F(8)$ & $B(2)$ & $1.41(1)$ \\
\hline$C(1)$ & $C(2)$ & $1.39(1)$ & $C(1)$ & $C(6)$ & $1.41(1)$ \\
\hline$C(2)$ & $C(3)$ & $1.42(1)$ & $C(3)$ & $C(4)$ & $1.39(2)$ \\
\hline$C(4)$ & $C(5)$ & $1.39(1)$ & $C(5)$ & $C(6)$ & 1.42(1) \\
\hline$C(7)$ & $\mathrm{C}(8)$ & $1.36(1)$ & $C(7)$ & $C(14)$ & $1.49(1)$ \\
\hline$C(8)$ & $\mathrm{C}(9)$ & $1.52(1)$ & $C(9)$ & $C(10)$ & $1.55(1)$ \\
\hline$C(10)$ & $\mathrm{C}(11)$ & $1.48(1)$ & $C(11)$ & $\mathrm{C}(12)$ & $1.36(1)$ \\
\hline C(12) & $C(13)$ & $1.55(1)$ & $C(13)$ & $C(14)$ & $1.56(1)$ \\
\hline$C(15)$ & $C(16)$ & 1.41(1) & $C(15)$ & $C(20)$ & $1.39(1)$ \\
\hline$C(16)$ & $\mathrm{C}(17)$ & $1.38(1)$ & $\mathrm{C}(17)$ & $C(18)$ & $1.39(1)$ \\
\hline$C(18)$ & $\mathrm{C}(19)$ & $1.40(1)$ & $\mathrm{C}(19)$ & $C(20)$ & $1.39(1)$ \\
\hline $\mathrm{C}(21)$ & $\mathrm{C}(22)$ & $1.39(1)$ & $\mathrm{C}(21)$ & $\mathrm{C}(28)$ & $1.52(1)$ \\
\hline C(22) & $\mathrm{C}(23)$ & $1.48(2)$ & $\mathrm{C}(23)$ & $\mathrm{C}(24)$ & 1.53(1) \\
\hline $\mathrm{C}(24)$ & $C(25)$ & $1.52(1)$ & $\mathrm{C}(25)$ & $\mathrm{C}(26)$ & 1.33(1) \\
\hline$C(26)$ & $\mathrm{C}(27)$ & $1.48(1)$ & $\mathrm{C}(27)$ & $\mathrm{C}(28)$ & $1.54(2)$ \\
\hline C(29) & $\mathrm{C}(30)$ & $1.41(1)$ & $\mathrm{C}(29)$ & C(34) & 1.42(1) \\
\hline C(30) & $\mathrm{C}(31)$ & $1.39(1)$ & $\mathrm{C}(31)$ & $\mathrm{C}(32)$ & 1.34(2) \\
\hline C(32) & $\mathrm{C}(33)$ & $1.43(1)$ & $\mathrm{C}(33)$ & $\mathrm{C}(34)$ & $1.38(1)$ \\
\hline C(35) & $C(36)$ & $1.36(1)$ & $\mathrm{C}(35)$ & $\mathrm{C}(42)$ & 1.52(1) \\
\hline $\mathrm{C}(36)$ & $\mathrm{C}(37)$ & $1.50(1)$ & $\mathrm{C}(37)$ & C(38) & $1.53(1)$ \\
\hline C(38) & $\mathrm{C}(39)$ & $1.57(1)$ & $\mathrm{C}(39)$ & $\mathrm{C}(40)$ & 1.37(1) \\
\hline$C(40)$ & $\mathrm{C}(41)$ & $1.53(1)$ & $\mathrm{C}(41)$ & $\mathrm{C}(42)$ & $1.56(2)$ \\
\hline C(43) & $\mathrm{C}(44)$ & 1.41(1) & $\mathrm{C}(43)$ & $\mathrm{C}(48)$ & 1.37(2) \\
\hline $\mathrm{C}(44)$ & $\mathrm{C}(45)$ & 1.41(1) & $\mathrm{C}(45)$ & $\mathrm{C}(46)$ & 1.34(2) \\
\hline$C(46)$ & $\mathrm{C}(47)$ & $1.38(2)$ & $\mathrm{C}(47)$ & $\mathrm{C}(48)$ & 1.42(1) \\
\hline $\mathrm{C}(49)$ & $C(50)$ & $1.37(2)$ & $\mathrm{C}(49)$ & $C(56)$ & 1.51(1) \\
\hline$C(50)$ & $C(51)$ & $1.56(2)$ & $C(51)$ & $\mathrm{C}(52)$ & 1.52(2) \\
\hline C(52) & C(53) & $1.51(2)$ & $C(53)$ & $C(54)$ & 1.39(2) \\
\hline C(54) & C(55) & $1.49(2)$ & $C(55)$ & $C(56)$ & $1.53(2)$ \\
\hline
\end{tabular}


Table 4. Bond lengths involving hydrogens $(\AA)$

\begin{tabular}{|c|c|c|c|c|c|}
\hline atom & atom & distance & atom & atom & distance \\
\hline$O(1)$ & $\mathrm{H}(1)$ & $0.950(8)$ & $\mathrm{O}(2)$ & $H(36)$ & $0.950(9)$ \\
\hline$C(2)$ & $\mathrm{H}(2)$ & $0.95(1)$ & $C(3)$ & $\mathrm{H}(3)$ & $0.95(1)$ \\
\hline$C(4)$ & $\mathrm{H}(4)$ & $0.95(1)$ & $C(5)$ & $H(5)$ & $0.95(1)$ \\
\hline$C(6)$ & $H(6)$ & $0.95(1)$ & $C(7)$ & $\mathrm{H}(7)$ & $0.95(1)$ \\
\hline$C(8)$ & $\mathrm{H}(8)$ & $0.95(1)$ & $\mathrm{C}(9)$ & $\mathrm{H}(9)$ & $0.95(1)$ \\
\hline$C(9)$ & $H(10)$ & $0.95(1)$ & $C(10)$ & $H(11)$ & $0.95(1)$ \\
\hline$C(10)$ & $H(12)$ & $0.95(1)$ & $C(11)$ & $H(13)$ & $0.95(1)$ \\
\hline$C(12)$ & $H(14)$ & $0.95(1)$ & $C(13)$ & $H(15)$ & $0.95(1)$ \\
\hline$C(13)$ & $H(16)$ & $0.95(1)$ & $C(14)$ & $H(17)$ & $0.95(1)$ \\
\hline$C(14)$ & $H(18)$ & $0.95(1)$ & $C(16)$ & $H(19)$ & $0.95(1)$ \\
\hline$C(17)$ & $\mathrm{H}(20)$ & $0.95(1)$ & $C(18)$ & $H(21)$ & $0.95(1)$ \\
\hline$C(19)$ & $H(22)$ & $0.95(2)$ & $\mathrm{C}(20)$ & $H(23)$ & $0.95(1)$ \\
\hline$C(21)$ & $\mathrm{H}(24)$ & $0.95(1)$ & $C(22)$ & $\mathrm{H}(25)$ & $0.95(1)$ \\
\hline$C(23)$ & $H(26)$ & $0.95(1)$ & $C(23)$ & $\mathrm{H}(27)$ & $0.95(2)$ \\
\hline$C(24)$ & $\mathrm{H}(28)$ & $0.95(2)$ & $C(24)$ & $\mathrm{H}(29)$ & $0.95(1)$ \\
\hline$C(25)$ & $\mathrm{H}(30)$ & $0.95(1)$ & $C(26)$ & $\mathrm{H}(31)$ & $0.95(1)$ \\
\hline$C(27)$ & $\mathrm{H}(32)$ & $0.95(2)$ & $\mathrm{C}(27)$ & $\mathrm{H}(33)$ & $0.95(1)$ \\
\hline$C(28)$ & $\mathrm{H}(34)$ & $0.95(2)$ & $\mathrm{C}(28)$ & $H(35)$ & $0.95(2)$ \\
\hline$C(30)$ & $\mathrm{H}(37)$ & $0.95(1)$ & $\mathrm{C}(31)$ & $H(38)$ & $0.95(1)$ \\
\hline$C(32)$ & $\mathrm{H}(39)$ & $0.95(1)$ & $\mathrm{C}(33)$ & $\mathrm{H}(40)$ & $0.95(1)$ \\
\hline$C(34)$ & $\mathrm{H}(41)$ & $0.95(1)$ & $C(35)$ & $\mathrm{H}(42)$ & $0.95(1)$ \\
\hline$C(36)$ & $\mathrm{H}(43)$ & $0.95(1)$ & $\mathrm{C}(37)$ & $\mathrm{H}(44)$ & $0.95(2)$ \\
\hline $\mathrm{C}(37)$ & $\mathrm{H}(45)$ & $0.95(1)$ & $C(38)$ & $\mathrm{H}(46)$ & $0.95(1)$ \\
\hline$C(38)$ & $\mathrm{H}(47)$ & $0.95(1)$ & $\mathrm{C}(39)$ & $\mathrm{H}(48)$ & $0.95(1)$ \\
\hline $\mathrm{C}(40)$ & $\mathrm{H}(49)$ & $0.95(1)$ & $C(41)$ & $\mathrm{H}(50)$ & $0.95(1)$ \\
\hline $\mathrm{C}(41)$ & $\mathrm{H}(51)$ & $0.95(1)$ & $C(42)$ & $\mathrm{H}(52)$ & $0.95(1)$ \\
\hline $\mathrm{C}(42)$ & $\mathrm{H}(53)$ & $0.95(2)$ & $\mathrm{C}(44)$ & $\mathrm{H}(54)$ & $0.95(1)$ \\
\hline $\mathrm{C}(45)$ & $\mathrm{H}(55)$ & $0.95(2)$ & $C(46)$ & $\mathrm{H}(56)$ & $0.95(1)$ \\
\hline $\mathrm{C}(47)$ & $\mathrm{H}(57)$ & $0.95(2)$ & $\mathrm{C}(48)$ & $\mathrm{H}(58)$ & $0.95(1)$ \\
\hline $\mathrm{C}(49)$ & $\mathrm{H}(59)$ & $0.95(1)$ & $C(50)$ & $\mathrm{H}(60)$ & $0.95(2)$ \\
\hline $\mathrm{C}(51)$ & $\mathrm{H}(61)$ & $0.95(2)$ & $C(51)$ & $\mathrm{H}(62)$ & $0.95(2)$ \\
\hline $\mathrm{C}(52)$ & $\mathrm{H}(63)$ & $0.95(2)$ & $C(52)$ & $\mathrm{H}(64)$ & $0.95(2)$ \\
\hline C(53) & $\mathrm{H}(65)$ & $0.95(2)$ & $C(54)$ & $\mathrm{H}(66)$ & $0.95(1)$ \\
\hline C(55) & $\mathrm{H}(67)$ & $0.95(2)$ & $C(55)$ & $\mathrm{H}(68)$ & $0.95(3)$ \\
\hline$C(56)$ & $\mathrm{H}(69)$ & $0.95(1)$ & $C(56)$ & $\mathrm{H}(70)$ & $0.95(1)$ \\
\hline
\end{tabular}


Table 5. Bond angles ( $\left.{ }^{\circ}\right)$

\begin{tabular}{|c|c|c|c|c|c|c|c|}
\hline atom & atom & atom & angle & atom & atom & atom & angle \\
\hline $\mathrm{O}(1)$ & $\operatorname{Pt}(1)$ & $C(1)$ & $88.1(3)$ & $\mathrm{O}(1)$ & $\operatorname{Pt}(1)$ & $C(7)$ & $93.3(3)$ \\
\hline$O(1)$ & $\operatorname{Pt}(1)$ & $C(8)$ & $97.0(3)$ & $O(1)$ & $\operatorname{Pt}(1)$ & $C(11)$ & $159.9(3)$ \\
\hline $\mathrm{O}(1)$ & $\mathrm{Pt}(1)$ & $\mathrm{C}(12)$ & 162.6(3) & $C(1)$ & $\mathrm{Pt}(1)$ & $\mathrm{C}(7)$ & $165.6(4)$ \\
\hline$C(1)$ & $\mathrm{Pt}(1)$ & $C(8)$ & 159.6(3) & $C(1)$ & $\mathrm{Pt}(1)$ & $C(11)$ & $88.5(3)$ \\
\hline$C(1)$ & $\mathrm{Pt}(1)$ & $\mathrm{C}(12)$ & $92.8(4)$ & $C(7)$ & $\mathrm{Pt}(1)$ & $\mathrm{C}(8)$ & $34.2(3)$ \\
\hline $\mathrm{C}(7)$ & $\mathrm{Pt}(1)$ & $C(11)$ & $94.9(3)$ & $\mathrm{C}(7)$ & $\mathrm{Pt}(1)$ & $\mathrm{C}(12)$ & 81.6(4) \\
\hline$C(8)$ & $\mathrm{Pt}(1)$ & $\mathrm{C}(11)$ & 79.9(3) & $C(8)$ & $\mathrm{Pt}(1)$ & $\mathrm{C}(12)$ & $88.2(4)$ \\
\hline$C(11)$ & $\mathrm{Pt}(1)$ & $\mathrm{C}(12)$ & $37.5(4)$ & $\mathrm{O}(1)$ & $\mathrm{Pt}(2)$ & $C(15)$ & $90.3(3)$ \\
\hline $\mathrm{O}(1)$ & $\mathrm{Pt}(2)$ & $\mathrm{C}(21)$ & 158.2(3) & $\mathrm{O}(1)$ & $\mathrm{Pt}(2)$ & $\mathrm{C}(22)$ & 164.2(3) \\
\hline $\mathrm{O}(1)$ & $\mathrm{Pt}(2)$ & C(25) & $91.6(3)$ & $\mathrm{O}(1)$ & $\mathrm{Pt}(2)$ & $C(26)$ & $94.3(3)$ \\
\hline$C(15)$ & $\mathrm{Pt}(2)$ & $\mathrm{C}(21)$ & $89.4(4)$ & $C(15)$ & $\mathrm{Pt}(2)$ & $\mathrm{C}(22)$ & $91.6(4)$ \\
\hline$C(15)$ & $\mathrm{Pt}(2)$ & $\mathrm{C}(25)$ & 158.6(3) & $C(15)$ & $\mathrm{Pt}(2)$ & $C(26)$ & 166.3(3) \\
\hline $\mathrm{C}(21)$ & $\mathrm{Pt}(2)$ & $\mathrm{C}(22)$ & $37.5(3)$ & $C(21)$ & $\mathrm{Pt}(2)$ & $C(25)$ & $96.5(4)$ \\
\hline $\mathrm{C}(21)$ & $\mathrm{Pt}(2)$ & $C(26)$ & $81.6(3)$ & $C(22)$ & $\mathrm{Pt}(2)$ & $C(25)$ & $81.0(4)$ \\
\hline $\mathrm{C}(22)$ & $\mathrm{Pt}(2)$ & $C(26)$ & $87.5(4)$ & $C(25)$ & $\mathrm{Pt}(2)$ & $C(26)$ & $34.3(3)$ \\
\hline $\mathrm{O}(2)$ & $\mathrm{Pt}(3)$ & C(29) & $89.8(3)$ & $\mathrm{O}(2)$ & $\mathrm{Pt}(3)$ & $\mathrm{C}(35)$ & $90.3(3)$ \\
\hline $\mathrm{O}(2)$ & $\mathrm{Pt}(3)$ & $C(36)$ & $88.6(3)$ & $\mathrm{O}(2)$ & $\mathrm{Pt}(3)$ & $\mathrm{C}(39)$ & 165.8(3) \\
\hline $\mathrm{O}(2)$ & $\mathrm{Pt}(3)$ & $C(40)$ & $155.4(3)$ & $C(29)$ & $\operatorname{Pt}(3)$ & C(35) & 168.2(4) \\
\hline C(29) & $\mathrm{Pt}(3)$ & $C(36)$ & $157.7(4)$ & $C(29)$ & $\operatorname{Pt}(3)$ & $C(39)$ & $94.6(3)$ \\
\hline $\mathrm{C}(29)$ & $\mathrm{Pt}(3)$ & $\mathrm{C}(40)$ & $93.2(3)$ & $\mathrm{C}(35)$ & $\mathrm{Pt}(3)$ & $C(36)$ & $34.1(4)$ \\
\hline C(35) & $\mathrm{Pt}(3)$ & C(39) & $88.1(3)$ & $\mathrm{C}(35)$ & $\mathrm{Pt}(3)$ & $C(40)$ & $81.9(3)$ \\
\hline$C(36)$ & $\mathrm{Pt}(3)$ & C(39) & $82.2(3)$ & $C(36)$ & $\mathrm{Pt}(3)$ & $C(40)$ & $97.5(3)$ \\
\hline C(39) & $\mathrm{Pt}(3)$ & $C(40)$ & $37.9(4)$ & $\mathrm{O}(2)$ & $\mathrm{Pt}(4)$ & $\mathrm{C}(43)$ & $88.0(3)$ \\
\hline $\mathrm{O}(2)$ & $\mathrm{Pt}(4)$ & $\mathrm{C}(49)$ & $92.7(3)$ & $\mathrm{O}(2)$ & $\mathrm{Pt}(4)$ & $C(50)$ & $96.3(3)$ \\
\hline $\mathrm{O}(2)$ & $\mathrm{Pt}(4)$ & $C(53)$ & $163.6(4)$ & $\mathrm{O}(2)$ & $\mathrm{Pt}(4)$ & $C(54)$ & $158.4(3)$ \\
\hline$C(43)$ & $\mathrm{Pt}(4)$ & $\mathrm{C}(49)$ & $159.7(4)$ & $C(43)$ & $\mathrm{Pt}(4)$ & $C(50)$ & $164.8(4)$ \\
\hline$C(43)$ & $\mathrm{Pt}(4)$ & $C(53)$ & $90.0(5)$ & $C(43)$ & $\mathrm{Pt}(4)$ & $\mathrm{C}(54)$ & $91.6(4)$ \\
\hline $\mathrm{C}(49)$ & $\mathrm{Pt}(4)$ & $\mathrm{C}(50)$ & $35.1(4)$ & $\mathrm{C}(49)$ & $\mathrm{Pt}(4)$ & C(53) & $94.9(4)$ \\
\hline C(49) & $\mathrm{Pt}(4)$ & C(54) & $80.4(4)$ & C(50) & $\mathrm{Pt}(4)$ & C(53) & $81.8(5)$ \\
\hline$C(50)$ & $\mathrm{Pt}(4)$ & $\mathrm{C}(54)$ & $89.8(4)$ & C(53) & $\mathrm{Pt}(4)$ & C(54) & $38.0(4)$ \\
\hline $\mathrm{Pt}(1)$ & $\mathrm{O}(1)$ & $\mathrm{Pt}(2)$ & 118.6(3) & $\mathrm{Pt}(3)$ & $\mathrm{O}(2)$ & $\mathrm{Pt}(4)$ & $115.6(3)$ \\
\hline$C(2)$ & $C(1)$ & $C(6)$ & 118.9(8) & $\mathrm{C}(2)$ & $C(1)$ & $\mathrm{Pt}(1)$ & $121.1(7)$ \\
\hline$C(6)$ & $C(1)$ & $\operatorname{Pt}(1)$ & 120.0(6) & $\mathrm{C}(3)$ & $C(2)$ & $C(1)$ & $121.0(9)$ \\
\hline$C(4)$ & $C(3)$ & $C(2)$ & 119.9(9) & $\mathrm{C}(5)$ & C(4) & C(3) & $119.6(9)$ \\
\hline$C(6)$ & $C(5)$ & $C(4)$ & 120.4(9) & $C(1)$ & $C(6)$ & $\mathrm{C}(5)$ & $120.1(8)$ \\
\hline$C(8)$ & $\mathrm{C}(7)$ & $\mathrm{C}(14)$ & 127.4(9) & $\mathrm{C}(8)$ & $C(7)$ & $\mathrm{Pt}(1)$ & 74.2(5) \\
\hline$C(14)$ & $\mathrm{C}(7)$ & $\operatorname{Pt}(1)$ & 105.5(5) & $\mathrm{C}(9)$ & $\mathrm{C}(8)$ & $\mathrm{Pt}(1)$ & 108.6(5) \\
\hline
\end{tabular}


Table 5. Bond angles $\left({ }^{0}\right)$-- continued

\begin{tabular}{|c|c|c|c|c|c|c|c|}
\hline atom & atom & atom & angle & atom & atom & atom & angle \\
\hline $\mathrm{C}(9)$ & $C(8)$ & $C(7)$ & $123.2(9)$ & $C(7)$ & $C(8)$ & $\mathrm{Pt}(1)$ & $71.6(5)$ \\
\hline$C(10)$ & $C(9)$ & $C(8)$ & 111.8(8) & $C(11)$ & $C(10)$ & $\mathrm{C}(9)$ & $113.4(8)$ \\
\hline$C(12)$ & $C(11)$ & $\operatorname{Pt}(1)$ & $69.7(5)$ & $C(12)$ & $C(11)$ & $C(10)$ & $130.4(8)$ \\
\hline $\mathrm{Pt}(1)$ & $\mathrm{C}(11)$ & $C(10)$ & $109.4(6)$ & $C(13)$ & $C(12)$ & $\operatorname{Pt}(1)$ & 113.4(6) \\
\hline$C(13)$ & $C(12)$ & $C(11)$ & $122.3(9)$ & $C(11)$ & $C(12)$ & $\mathrm{Pt}(1)$ & $72.8(6)$ \\
\hline$C(14)$ & $C(13)$ & $C(12)$ & 113.6(8) & $C(7)$ & $C(14)$ & $C(13)$ & $113.1(7)$ \\
\hline$C(16)$ & $C(15)$ & $C(20)$ & $117.8(9)$ & $C(16)$ & $C(15)$ & $\mathrm{Pt}(2)$ & 119.4(7) \\
\hline$C(20)$ & $C(15)$ & $\operatorname{Pt}(2)$ & $122.6(6)$ & $C(17)$ & $C(16)$ & $C(15)$ & $120.7(9)$ \\
\hline$C(18)$ & $C(17)$ & $C(16)$ & $120.3(8)$ & $C(19)$ & $C(18)$ & $C(17)$ & $119(1)$ \\
\hline C(20) & $C(19)$ & $C(18)$ & $118(1)$ & $C(15)$ & $C(20)$ & $C(19)$ & $122.2(8)$ \\
\hline C(22) & $C(21)$ & C(28) & $125.4(9)$ & C(22) & $C(21)$ & $\mathrm{Pt}(2)$ & $71.3(5)$ \\
\hline$C(28)$ & $C(21)$ & $\operatorname{Pt}(2)$ & $108.1(6)$ & $C(23)$ & $C(22)$ & $\mathrm{Pt}(2)$ & $112.4(6)$ \\
\hline C(23) & $C(22)$ & $C(21)$ & $125.2(8)$ & $C(21)$ & $C(22)$ & $\mathrm{Pt}(2)$ & $71.2(5)$ \\
\hline$C(24)$ & $C(23)$ & $C(22)$ & $114.1(9)$ & $C(25)$ & $C(24)$ & $C(23)$ & $112.4(8)$ \\
\hline$C(26)$ & $C(25)$ & $\operatorname{Pt}(2)$ & $74.2(6)$ & $C(26)$ & $C(25)$ & $C(24)$ & $127.5(9)$ \\
\hline $\mathrm{Pt}(2)$ & $C(25)$ & $C(24)$ & $105.3(6)$ & C(27) & $C(26)$ & $\mathrm{Pt}(2)$ & $108.6(6)$ \\
\hline$C(27)$ & $\mathrm{C}(26)$ & $C(25)$ & $125.7(9)$ & $C(25)$ & $\mathrm{C}(26)$ & $\operatorname{Pt}(2)$ & $71.5(6)$ \\
\hline C(28) & $C(27)$ & $C(26)$ & $115.9(9)$ & $C(21)$ & $C(28)$ & $C(27)$ & $112.8(8)$ \\
\hline$C(30)$ & $C(29)$ & $C(34)$ & $117.0(7)$ & $C(30)$ & $C(29)$ & $\mathrm{Pt}(3)^{\prime}$ & $124.3(7)$ \\
\hline$C(34)$ & $C(29)$ & $\operatorname{Pt}(3)$ & 118.7(6) & $C(31)$ & $C(30)$ & $C(29)$ & $120.9(9)$ \\
\hline$C(32)$ & $C(31)$ & $C(30)$ & $123.1(9)$ & $\mathrm{C}(33)$ & $C(32)$ & $C(31)$ & $117.4(8)$ \\
\hline$C(34)$ & $C(33)$ & $C(32)$ & $121.4(9)$ & $\mathrm{C}(29)$ & $C(34)$ & $C(33)$ & $120.0(9)$ \\
\hline$C(36)$ & $C(35)$ & $C(42)$ & $126.6(8)$ & $\mathrm{C}(36)$ & $C(35)$ & $\mathrm{Pt}(3)^{\prime}$ & $71.9(5)$ \\
\hline$C(42)$ & $C(35)$ & $\operatorname{Pt}(3)$ & $108.8(6)$ & $C(37)$ & $C(36)$ & $\operatorname{Pt}(3)$ & $103.8(6)$ \\
\hline C(37) & $C(36)$ & $C(35)$ & $125.9(8)$ & $C(35)$ & $C(36)$ & $\mathrm{Pt}(3)$ & $74.0(5)$ \\
\hline$C(38)$ & $C(37)$ & $C(36)$ & $114.2(8)$ & C(39) & $C(38)$ & $C(37)$ & $112.7(7)$ \\
\hline C(40) & $C(39)$ & $\operatorname{Pt}(3)$ & $71.2(5)$ & $\mathrm{C}(40)$ & $\mathrm{C}(39)$ & $\mathrm{C}(38)$ & 123.8(8) \\
\hline $\mathrm{Pt}(3)$ & $\mathrm{C}(39)$ & $C(38)$ & $112.4(6)$ & $\mathrm{C}(41)$ & $C(40)$ & $\mathrm{Pt}(3)$ & 109.6(6) \\
\hline$C(41)$ & $C(40)$ & $C(39)$ & $124.7(8)$ & C(39) & $\mathrm{C}(40)$ & $\mathrm{Pt}(3)$ & $70.9(5)$ \\
\hline$C(42)$ & $\mathrm{C}(41)$ & $C(40)$ & $115.5(9)$ & $C(35)$ & $\mathrm{C}(42)$ & $C(41)$ & $112.6(8)$ \\
\hline$C(44)$ & $C(43)$ & $C(48)$ & 119.1(9) & $C(44)$ & $C(43)$ & $\mathrm{Pt}(4)^{\prime}$ & $120.3(7)$ \\
\hline$C(48)$ & $C(43)$ & $\operatorname{Pt}(4)$ & $120.6(7)$ & $C(45)$ & $C(44)$ & $C(43)$ & $119(1)$ \\
\hline C(46) & $C(45)$ & $C(44)$ & $121(1)$ & $C(47)$ & $C(46)$ & $C(45)$ & $120(1)$ \\
\hline C(48) & $C(47)$ & $C(46)$ & 119(1) & $C(43)$ & $\mathrm{C}(48)$ & $C(47)$ & $120(1)$ \\
\hline$C(50)$ & C(49) & $C(56)$ & $125.3(9)$ & $C(50)$ & C(49) & $\mathrm{Pt}(4)$ & $71.5(6)$ \\
\hline C(56) & C(49) & $\mathrm{Pt}(4)$ & $108.8(7)$ & $C(51)$ & $C(50)$ & $\mathrm{Pt}(4)$ & $106.9(7)$ \\
\hline$C(51)$ & $C(50)$ & C(49) & $122(1)$ & C(49) & $C(50)$ & $\operatorname{Pt}(4)$ & $73.4(6)$ \\
\hline C(52) & $C(51)$ & $C(50)$ & $115.2(9)$ & $C(53)$ & $C(52)$ & $C(51)$ & 111(1) \\
\hline C(54) & $\mathrm{C}(53)$ & $\mathrm{Pt}(4)$ & $72.0(6)$ & C(54) & $C(53)$ & $C(52)$ & 131(1) \\
\hline $\mathrm{Pt}(4)$ & $C(53)$ & $C(52)$ & $109.4(9)$ & $C(55)$ & C(54) & $\mathrm{Pt}(4)$ & $114.3(9)$ \\
\hline$C(55)$ & $C(54)$ & $C(53)$ & $125(1)$ & $C(53)$ & $C(54)$ & $\mathrm{Pt}(4)$ & $70.0(5)$ \\
\hline C(56) & $C(55)$ & $C(54)$ & $116(1)$ & $C(49)$ & $C(56)$ & $C(55)$ & $115(1)$ \\
\hline$F(1)$ & $B(1)$ & $F(2)$ & $110(1)$ & $F(1)$ & $\mathrm{B}(1)$ & $F(3)$ & $106(1)$ \\
\hline$F(1)$ & $\mathrm{B}(1)$ & $\mathrm{F}(4)$ & 117(1) & $F(2)$ & $\mathrm{B}(1)$ & $F(3)$ & 105(1) \\
\hline$F(2)$ & $\mathrm{B}(1)$ & $\mathrm{F}(4)$ & $113(1)$ & $F(3)$ & $\mathrm{B}(1)$ & $F(4)$ & $103(1)$ \\
\hline$F(5)$ & $\mathrm{B}(2)$ & $F(6)$ & $108.7(8)$ & $F(5)$ & $\mathrm{B}(2)$ & $F(7)$ & $108.0(8)$ \\
\hline$F(5)$ & $\mathrm{B}(2)$ & $\mathrm{F}(8)$ & $109.2(9)$ & $F(6)$ & $\mathrm{B}(2)$ & $F(7)$ & $110.0(9)$ \\
\hline$F(6)$ & $\mathrm{B}(2)$ & $\mathrm{F}(8)$ & $110.9(8)$ & $\mathrm{F}(7)$ & $\mathrm{B}(2)$ & $F(8)$ & $110.0(8)$ \\
\hline
\end{tabular}


Table 6. Bond angles involving hydrogens $\left(^{\circ}\right)$

\begin{tabular}{|c|c|c|c|c|c|c|c|}
\hline atom & atom & atom & angle & atom & atom & atom & angle \\
\hline$H(1)$ & $\mathrm{O}(1)$ & $\operatorname{Pt}(1)$ & $120.8(6)$ & $H(1)$ & $\mathrm{O}(1)$ & $\operatorname{Pt}(2)$ & $120.6(7)$ \\
\hline $\mathrm{H}(36)$ & $\mathrm{O}(2)$ & $\operatorname{Pt}(3)$ & $122.1(6)$ & $H(36)$ & $O(2)$ & $\mathrm{Pt}(4)$ & $122.3(5)$ \\
\hline$C(3)$ & $C(2)$ & $\mathrm{H}(2)$ & $119(1)$ & $\mathrm{H}(2)$ & $C(2)$ & $C(1)$ & $119(1)$ \\
\hline$C(4)$ & $C(3)$ & $H(3)$ & 119(1) & $H(3)$ & $C(3)$ & $C(2)$ & $120(1)$ \\
\hline$C(5)$ & $C(4)$ & $\mathrm{H}(4)$ & $120(1)$ & $\mathrm{H}(4)$ & $C(4)$ & $C(3)$ & $120(1)$ \\
\hline$C(6)$ & $C(5)$ & $H(5)$ & $119(1)$ & $H(5)$ & $C(5)$ & $C(4)$ & 119(1) \\
\hline $\mathrm{H}(6)$ & $C(6)$ & $C(1)$ & $120(1)$ & $H(6)$ & $C(6)$ & $C(5)$ & 119(1) \\
\hline $\mathrm{C}(8)$ & $C(7)$ & $\mathrm{H}(7)$ & $114(1)$ & $C(14)$ & $C(7)$ & $\mathrm{H}(7)$ & $113(1)$ \\
\hline $\mathrm{H}(7)$ & $C(7)$ & $\operatorname{Pt}(1)$ & $113.9(9)$ & $\mathrm{C}(9)$ & $C(8)$ & $\mathrm{H}(8)$ & $115(1)$ \\
\hline $\mathrm{H}(8)$ & $C(8)$ & $\operatorname{Pt}(1)$ & 114.7(9) & $\mathrm{H}(8)$ & $C(8)$ & $C(7)$ & $115(1)$ \\
\hline$C(10)$ & $C(9)$ & $\mathrm{H}(9)$ & $109(1)$ & $C(10)$ & $C(9)$ & $\mathrm{H}(10)$ & $108(1)$ \\
\hline$H(9)$ & $C(9)$ & $\mathrm{H}(10)$ & 109(1) & $\mathrm{H}(9)$ & $C(9)$ & $C(8)$ & $108(1)$ \\
\hline$H(10)$ & $C(9)$ & $\mathrm{C}(8)$ & 109(1) & $C(11)$ & $C(10)$ & $H(11)$ & $107(1)$ \\
\hline$C(11)$ & $C(10)$ & $\mathrm{H}(12)$ & 109(1) & $H(11)$ & $C(10)$ & $\mathrm{H}(12)$ & 109(1) \\
\hline$H(11)$ & $C(10)$ & $\mathrm{C}(9)$ & $108(1)$ & $H(12)$ & $C(10)$ & $\mathrm{C}(9)$ & $108(1)$ \\
\hline$C(12)$ & $C(11)$ & $\mathrm{H}(13)$ & $112(1)$ & $\mathrm{H}(13)$ & $C(11)$ & $\operatorname{Pt}(1)$ & $112.8(9)$ \\
\hline$H(13)$ & $C(11)$ & $C(10)$ & $112(1)$ & $C(13)$ & $C(12)$ & $\mathrm{H}(14)$ & $113(1)$ \\
\hline $\mathrm{H}(14)$ & $C(12)$ & $\mathrm{Pt}(1)$ & $113(1)$ & $\mathrm{H}(14)$ & $C(12)$ & $C(11)$ & $113(1)$ \\
\hline$C(14)$ & $C(13)$ & $\mathrm{H}(15)$ & $108.8(9)$ & $C(14)$ & $C(13)$ & $H(16)$ & $108.2(9)$ \\
\hline$H(15)$ & $C(13)$ & $\mathrm{H}(16)$ & $109(1)$ & $H(15)$ & $C(13)$ & $C(12)$ & $108(1)$ \\
\hline$H(16)$ & $C(13)$ & $C(12)$ & $108(1)$ & $\mathrm{H}(17)$ & $C(14)$ & $H(18)$ & 109(1) \\
\hline $\mathrm{H}(17)$ & $C(14)$ & $C(7)$ & $109(1)$ & $\mathrm{H}(17)$ & $C(14)$ & $C(13)$ & $108.5(9)$ \\
\hline $\mathrm{H}(18)$ & $C(14)$ & $C(7)$ & $107(1)$ & $\mathrm{H}(18)$ & $C(14)$ & $C(13)$ & $108(1)$ \\
\hline$C(17)$ & $C(16)$ & $\mathrm{H}(19)$ & $119(1)$ & $H(19)$ & $C(16)$ & $C(15)$ & $119(1)$ \\
\hline$C(18)$ & $C(17)$ & $\mathrm{H}(20)$ & $119(1)$ & $\mathrm{H}(20)$ & $C(17)$ & $C(16)$ & $119(1)$ \\
\hline$C(19)$ & $C(18)$ & $\mathrm{H}(21)$ & $120(1)$ & $\mathrm{H}(21)$ & $C(18)$ & $C(17)$ & $119(1)$ \\
\hline$C(20)$ & $C(19)$ & $\mathrm{H}(22)$ & $120(1)$ & $\mathrm{H}(22)$ & $C(19)$ & $C(18)$ & $120(1)$ \\
\hline$H(23)$ & $C(20)$ & $C(15)$ & $118(1)$ & $\mathrm{H}(23)$ & $C(20)$ & $C(19)$ & $119(1)$ \\
\hline $\mathrm{C}(22)$ & $\mathrm{C}(21)$ & $\mathrm{H}(24)$ & $114(1)$ & $\mathrm{C}(28)$ & $C(21)$ & $\mathrm{H}(24)$ & $113(1)$ \\
\hline $\mathrm{H}(24)$ & $C(21)$ & $\operatorname{Pt}(2)$ & $114.5(9)$ & $\mathrm{C}(23)$ & $\mathrm{C}(22)$ & $\mathrm{H}(25)$ & $113(1)$ \\
\hline $\mathrm{H}(25)$ & $\mathrm{C}(22)$ & $\mathrm{Pt}(2)$ & $113(1)$ & $\mathrm{H}(25)$ & C(22) & $\mathrm{C}(21)$ & $113(1)$ \\
\hline $\mathrm{C}(24)$ & $\mathrm{C}(23)$ & $\mathrm{H}(26)$ & $107(1)$ & $C(24)$ & $\mathrm{C}(23)$ & $\mathrm{H}(27)$ & $108(1)$ \\
\hline $\mathrm{H}(26)$ & $\mathrm{C}(23)$ & $\mathrm{H}(27)$ & $109(1)$ & $\mathrm{H}(26)$ & $\mathrm{C}(23)$ & $\mathrm{C}(22)$ & $107(1)$ \\
\hline $\mathrm{H}(27)$ & $\mathrm{C}(23)$ & $\mathrm{C}(22)$ & $109(1)$ & $C(25)$ & $\mathrm{C}(24)$ & $\mathrm{H}(28)$ & $108(1)$ \\
\hline C(25) & $C(24)$ & $\mathrm{H}(29)$ & $108(1)$ & $\mathrm{H}(28)$ & C(24) & $\mathrm{H}(29)$ & 109(1) \\
\hline $\mathrm{H}(28)$ & $C(24)$ & $\mathrm{C}(23)$ & $109(1)$ & $\mathrm{H}(29)$ & $\mathrm{C}(24)$ & $\mathrm{C}(23)$ & $107(1)$ \\
\hline$C(26)$ & $C(25)$ & $\mathrm{H}(30)$ & $114(1)$ & $\mathrm{H}(30)$ & C(25) & $\operatorname{Pt}(2)$ & $113(1)$ \\
\hline
\end{tabular}


Table 6. Bond angles involving hydrogens $\left(^{0}\right)$-- continued

\begin{tabular}{|c|c|c|c|c|c|c|c|}
\hline atom & atom & atom & angle & atom & atom & atom & angle \\
\hline$H(30)$ & $\mathrm{C}(25)$ & $C(24)$ & $113(1)$ & $C(27)$ & $\mathrm{C}(26)$ & $\mathrm{H}(31)$ & $113(1)$ \\
\hline $\mathrm{H}(31)$ & $C(26)$ & $\operatorname{Pt}(2)$ & $114(1)$ & $\mathrm{H}(31)$ & $C(26)$ & $C(25)$ & 114(1) \\
\hline$C(28)$ & $C(27)$ & $\mathrm{H}(32)$ & $107(1)$ & $\mathrm{C}(28)$ & $C(27)$ & $\mathrm{H}(33)$ & $108(1)$ \\
\hline $\mathrm{H}(32)$ & $\mathrm{C}(27)$ & $\mathrm{H}(33)$ & 109(1) & $\mathrm{H}(32)$ & $\mathrm{C}(27)$ & $\mathrm{C}(26)$ & $106(1)$ \\
\hline$H(33)$ & $\mathrm{C}(27)$ & $C(26)$ & $108(1)$ & $\mathrm{H}(34)$ & $\mathrm{C}(28)$ & $\mathrm{H}(35)$ & $109(1)$ \\
\hline$H(34)$ & $\mathrm{C}(28)$ & $C(21)$ & $108(1)$ & $\mathrm{H}(34)$ & $\mathrm{C}(28)$ & $\mathrm{C}(27)$ & $107(1)$ \\
\hline $\mathrm{H}(35)$ & $\mathrm{C}(28)$ & $\mathrm{C}(21)$ & $109(1)$ & $\mathrm{H}(35)$ & $\mathrm{C}(28)$ & $\mathrm{C}(27)$ & 109(1) \\
\hline$C(31)$ & $C(30)$ & $\mathrm{H}(37)$ & $120(1)$ & $\mathrm{H}(37)$ & $C(30)$ & C(29) & $118(1)$ \\
\hline$C(32)$ & $C(31)$ & $\mathrm{H}(38)$ & $118(1)$ & $\mathrm{H}(38)$ & $C(31)$ & $C(30)$ & $118(1)$ \\
\hline$C(33)$ & $C(32)$ & $\mathrm{H}(39)$ & $120(1)$ & $\mathrm{H}(39)$ & $C(32)$ & $C(31)$ & 121(1) \\
\hline$C(34)$ & $C(33)$ & $\mathrm{H}(40)$ & 119(1) & $\mathrm{H}(40)$ & $\mathrm{C}(33)$ & C(32) & 119(1) \\
\hline $\mathrm{H}(41)$ & $C(34)$ & $C(29)$ & $119.7(9)$ & $\mathrm{H}(41)$ & $C(34)$ & C(33) & $120(1)$ \\
\hline$C(36)$ & $C(35)$ & $\mathrm{H}(42)$ & $114(1)$ & $C(42)$ & $C(35)$ & $\mathrm{H}(42)$ & $113(1)$ \\
\hline $\mathrm{H}(42)$ & $C(35)$ & $\operatorname{Pt}(3)$ & $113.9(8)$ & $C(37)$ & $C(36)$ & $\mathrm{H}(43)$ & 114(1) \\
\hline $\mathrm{H}(43)$ & $C(36)$ & $\operatorname{Pt}(3)$ & 114.7(8) & $\mathrm{H}(43)$ & $C(36)$ & $C(35)$ & 114(1) \\
\hline$C(38)$ & $C(37)$ & $\mathrm{H}(44)$ & $108(1)$ & $C(38)$ & $C(37)$ & $\mathrm{H}(45)$ & $107(1)$ \\
\hline $\mathrm{H}(44)$ & $C(37)$ & $\mathrm{H}(45)$ & 109(1) & $\mathrm{H}(44)$ & $C(37)$ & $C(36)$ & $109(1)$ \\
\hline $\mathrm{H}(45)$ & $C(37)$ & $C(36)$ & $107(1)$ & C(39) & $C(38)$ & $\mathrm{H}(46)$ & $108(1)$ \\
\hline C(39) & $C(38)$ & $\mathrm{H}(47)$ & $107(1)$ & $\mathrm{H}(46)$ & $C(38)$ & $\mathrm{H}(47)$ & $109(1)$ \\
\hline $\mathrm{H}(46)$ & $C(38)$ & C(37) & $108(1)$ & $\mathrm{H}(47)$ & $C(38)$ & $C(37)$ & $108(1)$ \\
\hline C(40) & $C(39)$ & $\mathrm{H}(48)$ & $114(1)$ & $\mathrm{H}(48)$ & $C(39)$ & $\operatorname{Pt}(3)$ & $114.3(8)$ \\
\hline $\mathrm{H}(48)$ & C(39) & $C(38)$ & $113(1)$ & $C(41)$ & $C(40)$ & $\mathrm{H}(49)$ & $114(1)$ \\
\hline $\mathrm{H}(49)$ & $C(40)$ & $\mathrm{Pt}(3)$ & $114.4(8)$ & $\mathrm{H}(49)$ & $C(40)$ & C(39) & 114(1) \\
\hline C(42) & $C(41)$ & $H(50)$ & $108(1)$ & $\mathrm{C}(42)$ & $C(41)$ & $\mathrm{H}(51)$ & $107(1)$ \\
\hline$H(50)$ & $C(41)$ & $\mathrm{H}(51)$ & 109(1) & $H(50)$ & $C(41)$ & C(40) & $107(1)$ \\
\hline$H(51)$ & $C(41)$ & $C(40)$ & $107(1)$ & $H(52)$ & $C(42)$ & $H(53)$ & $109(1)$ \\
\hline$H(52)$ & C(42) & $C(35)$ & $107(1)$ & $H(52)$ & C(42) & $C(41)$ & $108(1)$ \\
\hline$H(53)$ & $C(42)$ & $C(35)$ & 109(1) & $H(53)$ & $C(42)$ & C(41) & $108(1)$ \\
\hline C(45) & C(44) & $\mathrm{H}(54)$ & $120(1)$ & $\mathrm{H}(54)$ & C(44) & $\mathrm{C}(43)$ & $120(1)$ \\
\hline C(46) & $C(45)$ & $\mathrm{H}(55)$ & $119(1)$ & $H(55)$ & $C(45)$ & C(44) & $119(1)$ \\
\hline C(47) & $C(46)$ & $H(56)$ & 119(1) & $H(56)$ & $C(46)$ & C(45) & $120(1)$ \\
\hline$C(48)$ & $C(47)$ & $\mathrm{H}(57)$ & $120(1)$ & $\mathrm{H}(57)$ & $C(47)$ & $C(46)$ & $120(1)$ \\
\hline$H(58)$ & $C(48)$ & $C(43)$ & $119(1)$ & $\mathrm{H}(58)$ & $C(48)$ & $C(47)$ & 119(1) \\
\hline$C(50)$ & $C(49)$ & $\mathrm{H}(59)$ & $114(1)$ & $C(56)$ & $C(49)$ & $\mathrm{H}(59)$ & 114(1) \\
\hline$H(59)$ & $C(49)$ & $\operatorname{Pt}(4)$ & $114.0(8)$ & $C(51)$ & $C(50)$ & $\mathrm{H}(60)$ & 115(1) \\
\hline$H(60)$ & $C(50)$ & $\mathrm{Pt}(4)$ & 115.3(9) & $\mathrm{H}(60)$ & $C(50)$ & C(49) & 114(1) \\
\hline$C(52)$ & $C(51)$ & $\mathrm{H}(61)$ & $108(1)$ & $C(52)$ & $C(51)$ & $\mathrm{H}(62)$ & 107(1) \\
\hline$H(61)$ & $C(51)$ & $\mathrm{H}(62)$ & 109(1) & $\mathrm{H}(61)$ & $C(51)$ & $C(50)$ & 107(1) \\
\hline$H(62)$ & $C(51)$ & $C(50)$ & 107(1) & $C(53)$ & C(52) & $\mathrm{H}(63)$ & 109(1) \\
\hline$C(53)$ & C(52) & $\mathrm{H}(64)$ & 109(1) & $\mathrm{H}(63)$ & C(52) & $\mathrm{H}(64)$ & 109(1) \\
\hline$H(63)$ & $C(52)$ & $C(51)$ & $108(1)$ & $\mathrm{H}(64)$ & $C(52)$ & $C(51)$ & $109(1)$ \\
\hline$C(54)$ & $C(53)$ & $\mathrm{H}(65)$ & 111(1) & $\mathrm{H}(65)$ & $C(53)$ & $\mathrm{Pt}(4)$ & $111.8(9)$ \\
\hline $\mathrm{H}(65)$ & $C(53)$ & $C(52)$ & 111(1) & $C(55)$ & C(54) & $\mathrm{H}(66)$ & $112(1)$ \\
\hline$H(66)$ & $C(54)$ & $\operatorname{Pt}(4)$ & $113.2(8)$ & $\mathrm{H}(66)$ & $C(54)$ & $C(53)$ & 114(1) \\
\hline$C(56)$ & $C(55)$ & $\mathrm{H}(67)$ & $106(1)$ & $C(56)$ & $C(55)$ & $\mathrm{H}(68)$ & $109(1)$ \\
\hline $\mathrm{H}(67)$ & C(55) & $\mathrm{H}(68)$ & 109(2) & $\mathrm{H}(67)$ & C(55) & $C(54)$ & $105(1)$ \\
\hline $\mathrm{H}(68)$ & C(55) & $C(54)$ & $109(1)$ & $\mathrm{H}(69)$ & $C(56)$ & $\mathrm{H}(70)$ & $109(1)$ \\
\hline$H(69)$ & $C(56)$ & $C(49)$ & $108(1)$ & $\mathrm{H}(69)$ & $C(56)$ & C(55) & $106(1)$ \\
\hline
\end{tabular}


S20

$\mathrm{H}(70) \quad \mathrm{C}(56) \quad \mathrm{C}(49)$

107(1)

$\mathrm{H}(70) \quad \mathrm{C}(56)$

C(55)

109(1) 